\title{
A three-dimensional analysis of primary failure of eruption in humans and mice
}

\author{
Nicha Tokavanich $^{1}$ | Aditi Gupta ${ }^{1}$ | Mizuki Nagata ${ }^{1}$ | Akira Takahashi $^{1}$ | \\ Yuki Matsushita $^{1}$ | Marilia Yatabe ${ }^{1}$ | Antonio Ruellas ${ }^{1}$ | Lucia Cevidanes ${ }^{1}$ | \\ Koutaro Maki $^{2}$ | Tetsutaro Yamaguchi ${ }^{2,3}$ (D) | Noriaki Ono ${ }^{1}$ | Wanida Ono ${ }^{1}$ (I)
}

\author{
${ }^{1}$ Department of Orthodontics and Pediatric \\ Dentistry, University of Michigan School of \\ Dentistry, Ann Arbor, MI, USA \\ ${ }^{2}$ Department of Orthodontics, School of \\ Dentistry, Showa University, Tokyo, Japan \\ ${ }^{3}$ Department of Oral Interdisciplinary, \\ Division of Orthodontics, Kanagawa Dental \\ University Graduate School of Dentistry, \\ Kanagawa, Japan \\ Correspondence \\ Wanida Ono, Department of Orthodontics \\ and Pediatric Dentistry, University of \\ Michigan School of Dentistry, Ann Arbor, \\ MI, USA. \\ Email:wono@umich.edu \\ Funding information \\ National Institute of Dental and Craniofacial \\ Research, Grant/Award Number: DE026666 \\ and DE027421; American Association of \\ Orthodontists Foundation
}

\begin{abstract}
Objectives: Primary failure of eruption (PFE) is a genetic disorder exhibiting the cessation of tooth eruption. Loss-of-function mutations in parathyroid hormone (PTH)/ parathyroid hormone-related peptide (PTHrP) receptor (PTH/PTHrP receptor, PPR) were reported as the underlying cause of this disorder in humans. We showed in a PFE mouse model that PTHrP-PPR signaling is responsible for normal dental follicle cell differentiation and tooth eruption. However, the mechanism underlying the eruption defect in PFE remains undefined. In this descriptive study, we aim to chronologically observe tooth eruption and root formation of mouse PFE molars through 3D microCT analyses.

Setting and Sample Population: Two individuals with PFE were recruited at Showa University. A mouse PFE model was generated by deleting PPR specifically in PTHrPexpressing dental follicle and divided into three groups, $P P R^{f l / f l} ; R 26 R^{\text {tdTomato/+}}$ (Control), PTHrP-creER;PPR ${ }^{f l /+} ; R 26 R^{\text {tdTomato/+ }}$ (cHet), and PTHrP-creER;PRR $R^{f l / f l} ; R 26 R^{\text {tdTomato/+ }}$ (cKO). Materials and Methods: Images from human PFE subjects were acquired by CBCT. All groups of mouse samples were studied at postnatal days 14, 25, 91, and 182 after a tamoxifen pulse at $\mathrm{P} 3$, and superimposition of $3 \mathrm{D}$ microCT images among three groups was rendered.

Results: Mouse and human PFE molars exhibited a similar presentation in the 3D CT analyses. The quantitative analysis in mice demonstrated a statistically significant decrease in the eruption height of cKO first and second molars compared to other groups after postnatal day 25 . Additionally, cKO molars demonstrated significantly shortened roots with dilacerations associated with the reduced interradicular bone height.

Conclusions: Mouse PFE molars erupt at a much slower rate compared to normal molars, associated with shortened and dilacerated roots and defective interradicular bones.
\end{abstract}




\section{KEYWORDS}

parathyroid hormone, parathyroid hormone-related peptide receptor type i, primary failure of eruption, receptor mutations, tooth eruption, tooth root formation

\section{1 | INTRODUCTION}

Tooth eruption is defined as a movement of a tooth from its intraosseous site to its functional position of occlusion (Massler \& Schour, 1941). The process consists of three stages: (a) pre-eruptive tooth movement, (b) eruptive tooth movement, and (c) posteruptive tooth movement (Nanci, ; Richman, 2019). It has been generally accepted that this process depends on the equilibrium between the spaces in the path of eruption created by the dental follicle and the eruption pressure from apical tooth root formation (Kjær, 2014). Dental follicle (DF) is a loose sac-like connective tissue surrounding the developing tooth (Honda, Imaizumi, Tsuchiya, \& Morsczeck, 2010). It comprises heterogeneous cell populations with two distinct roles during tooth eruption: activating and recruiting osteoclasts (Wise, 2009), and including mesenchymal progenitor cells that differentiate into periodontal ligament cells, osteoblasts that form alveolar cryptal bone, and cementoblasts of the acellular cementum (Takahashi et al., 2019).

In the eruptive stage of tooth eruption, the crucial genes involve colony-stimulating factor-one (CSF-1), receptor activator of nuclear factor- $\kappa \mathrm{B}$ ligand (RANKL) and osteoprotegerin (OPG), which are abundantly expressed by dental follicle mesenchymal cells. These factors play important roles in the recruitment of cells in the monocyte-macrophage lineage and osteoclastogenesis (Wise, Frazier-Bowers, \& D'Souza, 2002). Therefore, in this stage, DF facilitates tooth eruption by activating osteoclasts and subsequently clearing the eruption pathway for unerupted teeth. However, in the later phase of eruptive stage, the formation of tooth root and periodontium plays important roles in tooth eruption. Parathyroid hormone-related protein (PTHrP) and parathyroid hormone/PTHrP receptor (PPR) autocrine signaling are essential for regulating tooth eruption and tooth formation at this stage. PTHrP and PPR are highly expressed in a population of DF cells. Thus, dental follicle cells play critical dual roles throughout the process of tooth eruption (Nagata, Ono, \& Ono, 2019).

Disturbance in tooth eruption is mostly caused by local factors and less frequently found in conjunction with systemic disease or syndrome (Raghoebar, Boering, Vissink, \& Stegenga, 1991). Primary failure of eruption (PFE) is a rare autosomal dominant non-syndromic disorder with a prevalence of $0.06 \%$ (Baccetti, 2000). It is defined as incomplete eruption of initially non-ankylosed tooth despite the presence of a clear eruption pathway, which results in a posterior unilateral or bilateral open bite. PFE can affect both primary and permanent teeth, and most commonly affects posterior teeth (Proffit \& Vig, 1981). Clinically, two types of PFE have been defined by Frazier-Bowers et al. Type I PFE demonstrates lack of an eruption potential in all affected teeth, while type II PFE demonstrates a much milder presentation, in which the teeth distal to the most mesial affected teeth merely show an open bite condition (Frazier-Bowers, Koehler, Ackerman, \& Proffit, 2007). Treatment options for these conditions are limited, as the teeth affected by PFE do not respond to orthodontic forces; instead, they are likely to become ankylosed upon orthodontic intervention (Proffit \& Frazier-Bowers, 2009). Therefore, the accurate initial diagnosis and identification of the cause of posterior open bite prior to treatment is essential; to this end, it is important to understand the mechanism underlying this disease process.

Loss-of-function mutations in Parathyroid hormone receptor 1 (PTHR1, also known as PTH/PTHrP receptor, PPR) are associated with PFE in humans (Decker et al., 2008; Frazier-Bowers et al., 2014; Frazier-Bowers, Simmons, Wright, Proffit, \& Ackerman, 2010; Grippaudo, Cafiero, D'Apolito, Ricci, \& Frazier-Bowers, 2018; Jelani et al., 2016; Risom et al., 2013; Roth et al., 2014; Yamaguchi et al., 2011). PTHR1 is a G protein-coupled receptor that binds to parathyroid hormone (PTH) and parathyroid hormone-related peptide (PTHrP) in an equivalent affinity (Dean, Vilardaga, Potts, \& Gardella, 2008; Jüppner et al., 1991). During tooth development, PTHR1 is expressed in the dental mesenchyme and in the alveolar bone in close proximity to tooth buds, while PTHrP is highly expressed in the dental follicle (Ono, Sakagami, Nishimori, Ono, \& Kronenberg, 2016). Autocrine regulation of PTHrP and its receptor PPR is critical during differentiation of dental follicle cells. It allows proper formation of the periodontal attachment apparatus that promotes tooth root formation and eruption. In the absence of the PPR signaling in $\mathrm{PTHrP}^{+}$dental follicle cells, molars developed tooth root malformation associated with under-eruption, representing a mouse model of PFE (Takahashi et al., 2019).

A twenty-year follow-up of a familial PFE case shows that if normal eruption and alveolar bone development is disrupted before or during the puberty, tooth submergence becomes progressive. Subsequently, the earlier the onset of the disease was, the more severe the open bite becomes. Furthermore, open bite aggravates in the adulthood compared to the puberty, when the PFE becomes pronounced sufficient for diagnosis (Kanno, Oliveira, Garcia, Roth, \& Weber, 2017). However, due to the rarity of the disorder, how this eruption disorder develops over time remains incompletely understood. To further investigate how PFE develops chronologically, we utilized the mouse model of PFE that exhibits failure of eruption and truncated roots in a similar way to human PFE conditions. The objective of our study is to chronologically observe the abnormality of this phenotype using 3D microCT analyses.

\section{2 | MATERIALS AND METHODS}

\section{1 | Human subjects}

This patient's sample study was approved by the Ethics Committee of Showa University (IRB approval number: human genome/gene 
analysis ethics subcommittee \#118). Two individuals with PFE (Patient 1:15 y.o. male, Patient 2:29 y.o. female) were diagnosed and recruited from the Showa University Dental Hospital. Additional details about these PFE individuals have been described previously (Yamaguchi et al., 2011). Screening of PTH1R was performed by direct sequencing of PCR products covering the PTH1R coding exons (exon 3 to 16) and the respective intron-exon boundaries. Images were acquired using a cone-beam X-ray CT system for dental and maxillofacial use (CB MercuRay; Hitachi Medico Technology). The scanning conditions were $100 \mathrm{kVp}, 10 \mathrm{~mA}$, F-mode 512 slices/scan (slice width: $377 \mathrm{~mm}$ ), and 9.6-s acquisition time.

\section{2 | Three-dimensional image analysis for patient's sample study}

The original DICOM files (baseline) were converted to de-identified files in gipl.gz format using the ITK-SNAP open-source software (Cevidanes, Styner, \& Proffit, 2009). Additional details about the 3D image analysis have been described previously (Ruellas, Yatabe, et al., 2016). Briefly, the DICOM data for each patient were converted to de-identified files in gipl.gz format using ITK-SNAP open-source software. The initial step was to create a head orientation matrix for overlay; due to bilateral mandibular asymmetry, it was determined to orient the images based on the cranial base. A three-dimensional volumetric label map a.k.a. segmentation was constructed for each patient, wherein the cranial base, maxilla, mandible, and teeth were marked as one single label map using ITK-SNAP program. This full-face segmentation was used to create a surface model for the 3D label map using model maker tool in Slicer software. The full-face surface model was then oriented (Ruellas, Tonello, et al., 2016) via three planes in the 3D coordinate system-Frankfort horizontal, mid-sagittal and transporionic using following reference points. The mid-sagittal plane was determined by glabella and crista galli, the Frankfort horizontal plane by right, left porion and left orbitale; and the transporionic plane by a line passing through right and left porion perpendicular to the Frankfort horizontal plane. An orientation matrix was thus created for this head orientation using the transforms tool in Slicer, and this matrix was applied to the original scan. Then, all subsequent patient segmentations and surface models were oriented. Next, three-dimensional volumetric label maps a.k.a. segmentations were constructed for each patient, wherein the maxilla, mandible and teeth were separate label maps using ITK-SNAP program. Two such separate segmentation files were created to label hemisections of mandible for the affected and unaffected side. The semi-automatic segmentations used active contour method to define boundaries of label maps using image gray level and intensity. These segmentations were then converted to 3D surface models in Slicer software. The original files (scan, segmentation and surface models) were mirrored to allow the superimposition and the evaluation of the differences between affected and unaffected sides. Finally, the original and mirrored files were registered using voxel-based registration in Slicer software. The regional maxillary and mandibular registrations were performed to visually evaluate differences between affected and unaffected sides.

\section{3 | Mice}

PTHrP-creER bacterial artificial chromosome (BAC) mice have been established and described previously (Mizuhashi et al., 2018). PPRfloxed (Kobayashi et al., 2002) and Rosa26-CAG-loxP-stop-loxPtdTomato (Ai14: R26R-tdTomato, JAX007914) mice were obtained from the Jackson laboratory. All procedures were performed under the Guidelines for the Care and Use of Laboratory Animals approved by the University of Michigan's Institutional Animal Care and Use Committee (IACUC), protocol 7,000 (Ono). The mouse PFE model was created by crossing PTHrP-creER and PPR-floxed mice, generating three corresponding groups including $P P R^{f l / f l} ; R 26 R^{\text {tdTomato/+ }}$ (Control), PTHrP-creER; PPR ${ }^{\text {fl/t+}} ; R 26 R^{\text {tdTomato/+ }}$ (cHet) and PTHrP-creER; $P R R^{\text {fl/fl }} ; R 26 R^{\text {tdTomato/+ }}$ (cKO) mice(Takahashi et al., 2019). (Fig S1). The DNA extraction of mouse tail biopsies was done using a HotShot protocol, which involved incubation of the tail sample at $95^{\circ} \mathrm{C}$ for $30 \mathrm{~min}$ in an alkaline lysis reagent followed by neutralization. PCRbased genotyping was performed using a standard protocol (GoTaq Green Master Mix, Promega, and Nexus X2, Eppendorf). Mice were euthanized by overdosage of carbon dioxide or decapitation under inhalation anesthesia in a drop jar (Fluriso, Isoflurane USP, VetOne).

\section{4 | Tamoxifen}

Tamoxifen (Sigma T5648) was mixed and completely dissolved in $100 \%$ ethanol. A proper volume of sunflower seed oil (Sigma S5007) was added and thoroughly mixed to the tamoxifen-ethanol mixture afterward. The tamoxifen-ethanol-oil mixture was incubated at $60^{\circ} \mathrm{C}$ in a chemical hood until the ethanol evaporated completely. The final mixture was stored at room temperature until use.

\section{5 | Immunohistochemistry}

Samples were dissected and fixed in 4\% paraformaldehyde overnight. Then, mandibles were decalcified in 15\% EDTA for 1 day. Decalcified mandibles were cryoprotected in 30\% sucrose/PBS solutions overnight and then kept in 30\% sucrose/PBS:OCT (1:1) solutions. Subsequently, samples were embedded in an OCT compound (Tissue-Tek, Sakura) under a stereomicroscope (Nikon SMZ-800), which was then solidified on a sheet of dry ice. Embedded samples were cryosectioned at $16 \mu \mathrm{m}$ by a cryostat (Leica CM1850) and transferred to positively charged glass slides (Fisherbrand ColorFrost Plus). After stored in $-30^{\circ} \mathrm{C}$, sections were postfixed in $4 \%$ paraformaldehyde for 20 min. For staining, sections were permeabilized with $0.25 \%$ Triton-X/TBS for $30 \mathrm{~min}$, blocked with $3 \%$ BSA/TBST for 30 min, incubated with anti-PPR rabbit antibody (1:200, AssaybioTech G220) overnight at $4^{\circ} \mathrm{C}$, and incubated 
with donkey anti-rabbit secondary antibody conjugated with Alexa Flour 488 (A21206) for 3 hr. Lastly, sections were stained with DAPI (4',6-diamidino-2-phenylindole, $5 \mu \mathrm{g} / \mathrm{ml}$, Invitrogen D1306) prior to imaging.

\section{6 | Three-dimensional micro-computed tomography analysis of mouse samples}

Mandibles including molars and incisors were placed in a $19 \mathrm{~mm}$ diameter specimen holder and scanned as DICOM files using a microCT system ( $\mu \mathrm{CT} 100$ Scanco Medical) with voxel size $12 \mu \mathrm{m}, 70 \mathrm{kVp}, 114 \mu \mathrm{A}$ $0.5 \mathrm{~mm}$ AL filter, and integration time $500 \mathrm{~ms}$ scan settings. The DICOM files were converted into "Guys Image Processing Lab (gipl.gz)" files using ITK-SNAP open-source software. Three-dimensional volumetric label maps, a.k.a segmentation, of mouse mandible were created with ITK-SNAP software. Three-dimensional volumetric label maps of each sample were created wherein the mandible: incisor and three molars were labeled separately using active contour method to define boundaries of label maps of each tooth according to image gray leve and intensity. These segmentations were then converted to 3D surface models in Slicer open-source software. Using the Q3DC tool in slicer open-source software, fiducials were placed on the mandibular first molar, second molar, and inferior border of the mandible to determine hard tissue reference points. Fiducials were placed on the Menton (the most inferior point of border of mandible). For the molars, fiducials were placed on the mesial and distal root tips; the most concave point in the furcations; the most convex point on the buccal, lingual, mesial, and distal surfaces; as well as the cusp tip of mesiobuccal, distobuccal, and mesiolingual cusp tips. These landmarks were used in first, second, and third molars. Quantitative assessments were made by measuring the linear distance between fudicials in Slicer. To compare eruption height, 3D measurement between Menton and inferior-most point on the first molar root was compared between Control, cHet, and cKO samples. Another option to determine the under-eruption was to calculate 3D measurement between Menton and molar cusp tips. For crown morphology, the measurement between the mesiodistal and buccolingual width of the first mandibular molar was made and compared among three groups. To assess the root length, the distance between the cusp tip and molar root was measured and compared between samples. The interradicular bone was analyzed by measuring the distance between the midpoint of a line connecting the inferior point on mesial and distal root, to the fiducial in the furcation. For visualization of semi-transparent superimpositions, the surface models for littermate knockout and Het samples overlays were aligned using the transforms tool in Slicer open-source software. Inferior border of the mandible and condylar head were used as reference for the superimposition.

\subsection{Statistical analysis}

One-way ANOVA followed by Mann-Whitney's $U$ test was performed in order to compare means between three groups of samples.
In addition, linear regression was conducted to observe the change of the variables, including eruption height, root length, and crown width, over time and the slopes were compared. A $p$ value of $<0.05$ was considered significant.

\section{3 | RESULTS}

\subsection{Human PFE molars are associated with tooth root anomalies including truncation and dilacerations}

Primary failure of eruption (PFE) is the only known genetic condition that exclusively affects tooth eruption in humans. Tooth root phenotypes associated with PFE have not been documented. We reanalyzed two unrelated individuals diagnosed with PFE (Yamaguchi et al., 2011) using three-dimensional mandibular and maxillary registration, wherein cone-beam computed tomography (CBCT) images were analyzed using the surface-rendering ITK-SNAP and Slicer software programs (Ruellas, Yatabe, et al., 2016). Patient 1 was a familial PFE individual whose preceding two generations on the maternal side exhibited features of PFE, with clinical manifestation of a unilateral abnormality in the mandibular posterior segment (Figure 1a). Patient 2 was a sporadic PFE individual whose parents did not have clinical record, with clinical manifestation of a bilateral posterior abnormality (Figure $1 \mathrm{~b}$ ). Both patients carried a missense mutation in exon 6 of the PTH1R gene. We superimposed the right and left halves of the mandible using the cranial base as a reference structure. The PFE individuals, especially Patient 1 , exhibited an asymmetry in the mandibular body (Figure 1a, b, arrows). Importantly, both patients presented more pronounced PFE phenotypes on one side of the first molars (Figure 1a,b, yellow arrowheads). We next superimposed the right and left first molars using the crown as a reference structure. Tooth root anomalies of the severely affected molars were more pronounced, including dilacerations (curved roots, blue arrowheads) and truncation (short roots, green arrowheads). Therefore, the tooth eruption phenotype in PFE individuals occurs in the setting of tooth root anomalies in the mandibular first molars. Our finding is consistent with the cohort study which reported that 35 percent of human PFE patients exhibited tooth root anomaly (Pilz et al., 2014).

\subsection{Mouse PFE molars continue to erupt but at a much slower rate}

Dental follicle (DF) progenitor cells expressing parathyroid hormone-related protein (PTHrP) contribute to the formation of cementum, periodontal ligament, and alveolar bone cells. In this study, the eruption height, root length, and interradicular bone height were measured as the outcome of mouse PFE conditions. Littermate triple transgenic mice with three corresponding genotypes-Control (PPR $\left.{ }^{\mathrm{fl} / \mathrm{fl}}\right)$, (DF-PPR) cHet $\left(P T H r P-c r e E R ; P P R^{\mathrm{fl} /+}\right)$ and (DF-PPR) cKO (PTHrP-creER; $P P R^{\mathrm{fl} / \mathrm{fl})}$-were pulsed with a single 


\section{Mandible - Superimposed at the cranial base}
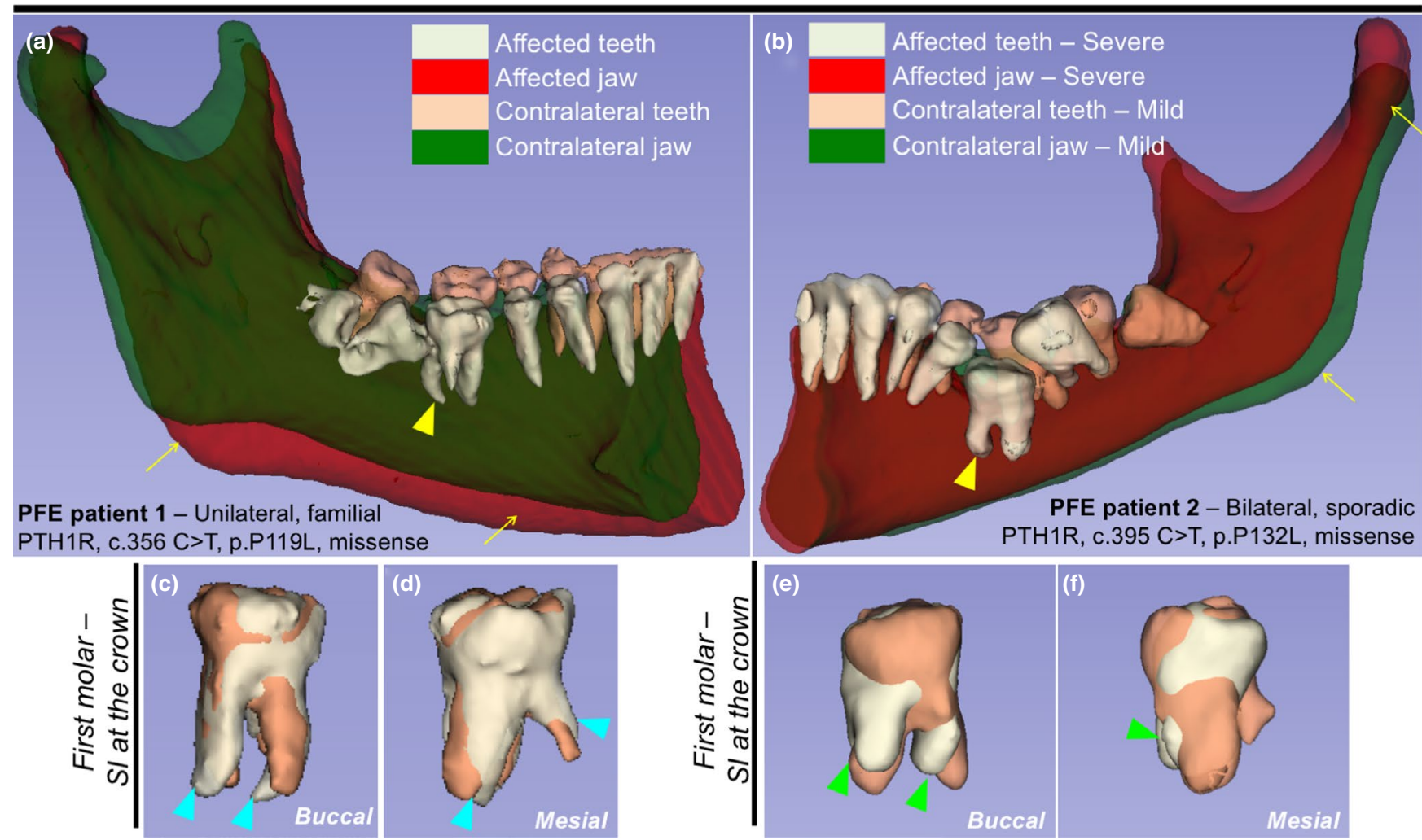

(e)
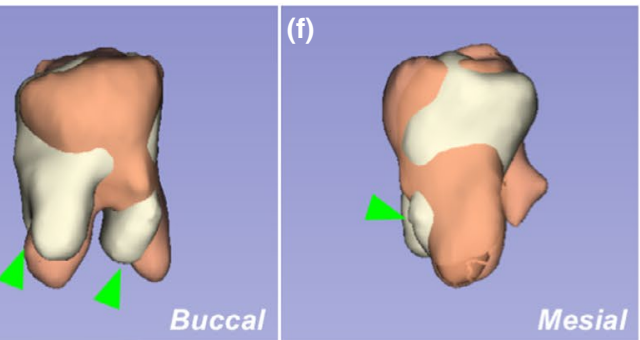

FIGURE 1 Human primary failure of eruption (PFE) molars are associated with tooth root anomalies including truncation and dilacerations. (a,b) 3D surface model overlays: mandibular superimposition of registered right and left hemisections (original and mirrored) within the same patient. Composite 3D computed tomography (CT) images of the mandible, views from the lingual side. (a) Registration was done using the cranial base. Familial PFE patient with unilateral posterior abnormality, PTH1R, c.356 C > T, p.P119L missense mutation. (b) Regional registration was done using the body of the mandible as reference. Sporadic PFE patient with bilateral posterior abnormality, PTH1R, c.395 C > T, p.P132L, missense mutation. Light green and red: teeth and jaw on the affected/severe mandibular hemisection, respectively. Beige and green: teeth and jaw on the contralateral/mild side, respectively. Yellow arrows: asymmetry of the mandibular body, yellow arrowheads: first molars associated with pronounced PFE phenotypes and tooth root anomalies. (c-f) Composite 3D surface model overlay of the mandibular first molars, views from the buccal side (c,e) and the mesial side (d, f). Bilateral first molars were superimposed on the crown. Light green: teeth on the affected/severe side, beige: teeth on the contralateral/mild side. Blue arrowheads: dilacerations (curved roots), green arrowheads: truncation (short roots) associated with dilacerations

dose of tamoxifen $(0.25 \mathrm{mg})$ at postnatal day 3. First, we performed immunohistochemical staining for PPR using an anti-PPR antibody (G220) to illustrate its expression levels in $\mathrm{PTHrP}^{+}$dental follicle cells. Overall, PPR expression was reduced in CKO molars compared to Control molars both at P8 and P18, as exemplified by the reduction in green signals (Figure S2). Furthermore, the reduction in yellow signals in cKO molars demonstrated that PPR expression was reduced in descendants of $\mathrm{PTHrP}^{+}$dental follicle cell at $\mathrm{P} 8$, as well as in cementoblasts and osteoblasts derived from $\mathrm{PTHrP}^{+}$ dental follicle cells at P18 (Figure S2). These data were consistent with our previous bulk RNA-seq finding that a significant reduction of reads occurred specifically in the second exon of Pth1r in PPRdeficient PTHrP ${ }^{+}$dental follicle cells, causing loss of functional PPR (Takahashi et al., 2019).

The 3D microCT data of PPR cKO mandibles were then compared with those of Control and cHet. The data were collected at 4 time points to observe the progression of the condition: (a) half root formation at postnatal day (P) 14, (b) completion of molar root formation and tooth eruption at P25, (c) fully grown mice at P91, and (d) middle-aged mice at P182. Similar to human PFE conditions, PPR cKO molars exhibited delayed tooth eruption, tooth root anomalies, and defective interradicular bones (Cohen-Lévy \& Cohen, 2015; Deffrennes \& Cohen-Lévy, 2015; Pilz et al., 2014; Figure 2). The statistical analysis revealed that the eruption height of cKO first and second molars (Figure 2i,j red line) was significantly lower than that of Control and cHet molars (Figure 2i,j black and blue line, respectively), starting from P25 to P182. The linear regression analysis showed no significant difference in all samples; however, in the first molar regression graph, there was a tendency that the difference in the height among CKO and other two groups increased over time, while in the second molar graph, the difference remained constant over time (Figure 2i,j). These results indicate that the PFE molars continue to erupt even after the roots are fully formed, but at a much slower rate than that of Control and cHet molars. This results in a much more pronounced defective tooth eruption phenotype especially in the first molars. 


\section{TAM injection at P3}
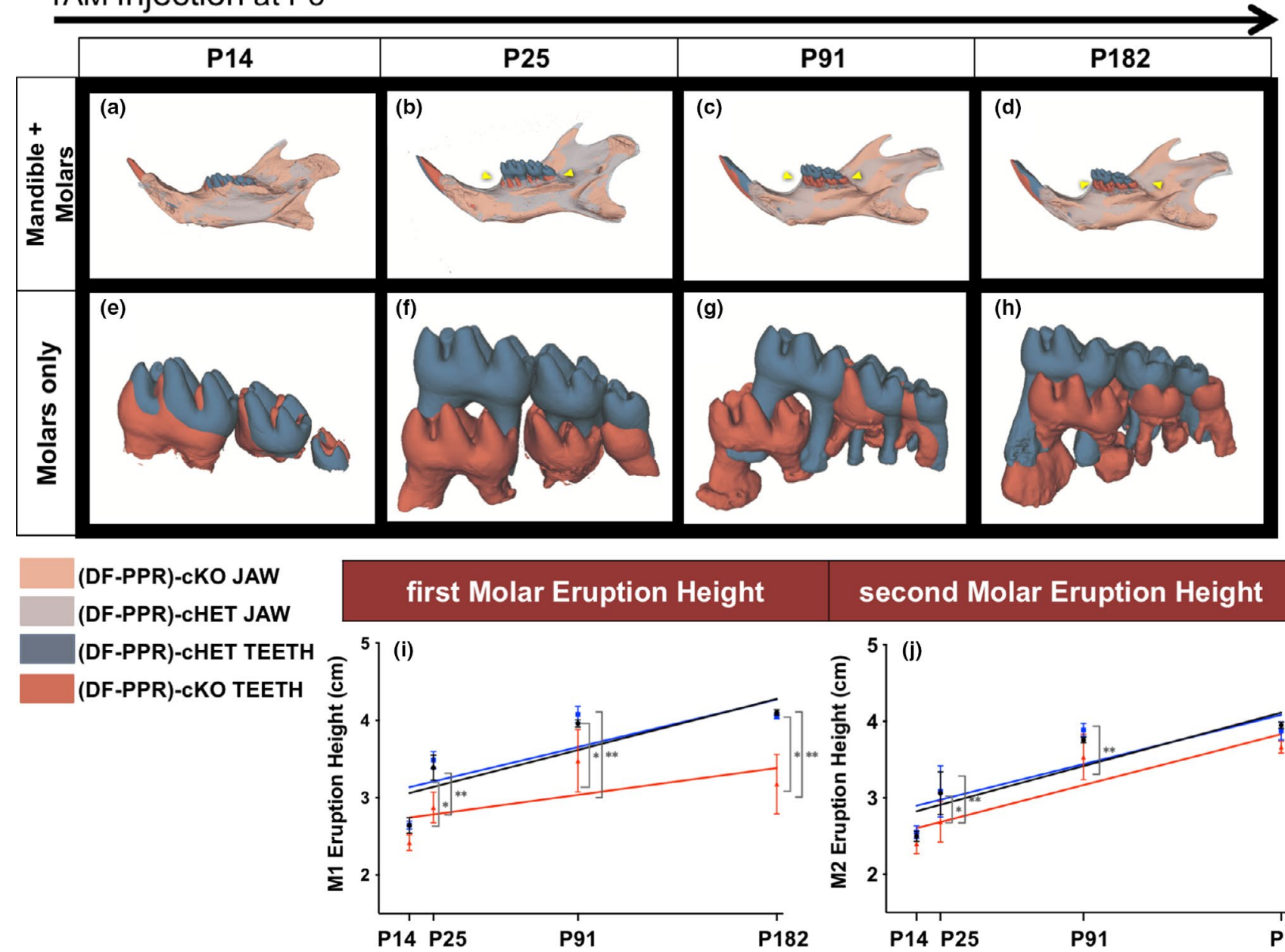

second Molar Eruption Height

FIGURE 2 Mouse primary failure of eruption (PFE) molars continue to erupt but at a much slower rate. (a-h) 3D surface model overlays: superimposition of registered dental follicle-specific (DF-PPR) cHet and (DF-PPR) cKO mandibles using mandibular border as a reference structure. At P14 (a,e), P25 (b,f), P91 (c,g) and P182 (d,h) after tamoxifen pulse at P3. Composite 3D computed tomography (CT) images of the mandible, views from the lingual side. Gray and dark gray: jaw and teeth of (DF-PPR) cHet mandible, respectively. Beige and red: jaw and teeth of (DF-PPR) cKO mandible, respectively. Yellow arrowhead: pronounced PFE phenotypes in (DF-PPR) cKO. (i,j) Quantitative 3D- $\mu$ CT analysis. Eruption height of first molars (M1) (i) and second molars (M2) (j) measured on 3D- $\mu \mathrm{CT}$ images. Colored lines: regression line, black: Control, blue: (DF-PPR) cHet, red: (DF-PPR) cKO. At P14 $n=5$, P2 $n=6$, P91 and P182 $n=4{ }^{* *} p<.01,{ }^{*} p<.05$, One-way ANOVA followed by Mann-Whitney's $U$ test. All data are represented as mean $\pm S D$

\subsection{Mouse PFE molars show shorter and dilacerated roots}

We further analyzed the length of molar roots in mouse PFE conditions (Figure 3). Statistical analysis revealed a significant reduction in the root length in the cKO first molars compared to Control and $\mathrm{cHet}$ molars, starting from P25; no significant difference was found at P14. No significant difference was noted between the regression slope of cKO (Figure $3 \mathrm{~g}$ red line) and other groups (Figure $3 \mathrm{~g}$ black-Control, and blue-cHet); however, the difference between in the cKO root length and those of other two groups tended to rise over time (Figure 3g). The superimposition analysis showed that cKO molars exhibited truncated and dilacerated roots at all time points except P14 (Figure 3a-d'"). No significant difference was noted in the crown morphology between cKO and other groups (Figure 3e,3f). These data suggested that the PPR deletion in PTHrP ${ }^{+}$dental follicle cells disrupts the formation of root in the later stage, which includes the formation of the apical half of the root, periodontal attachment, and surrounding alveolar bone. Subsequently, the emergence stage of eruption was interrupted.

\section{4 | Mouse PFE molars show defective cryptal bone formation}

Lastly, we measured the height of the interradicular bone at P182 (Figure 4). The interradicular bone heights of all molars were measured from axial section. The interradicular bone height was significantly decreased in cKO first and second molars, but not in third molars, compared to those of other two groups (Figure 4a,b). These data suggest defective bone formation surrounding the tooth root structure in mouse PFE molars (Figure 4c,d red-cKO, black-Control, and blue-cHet). 


\section{TAM injection at P3}

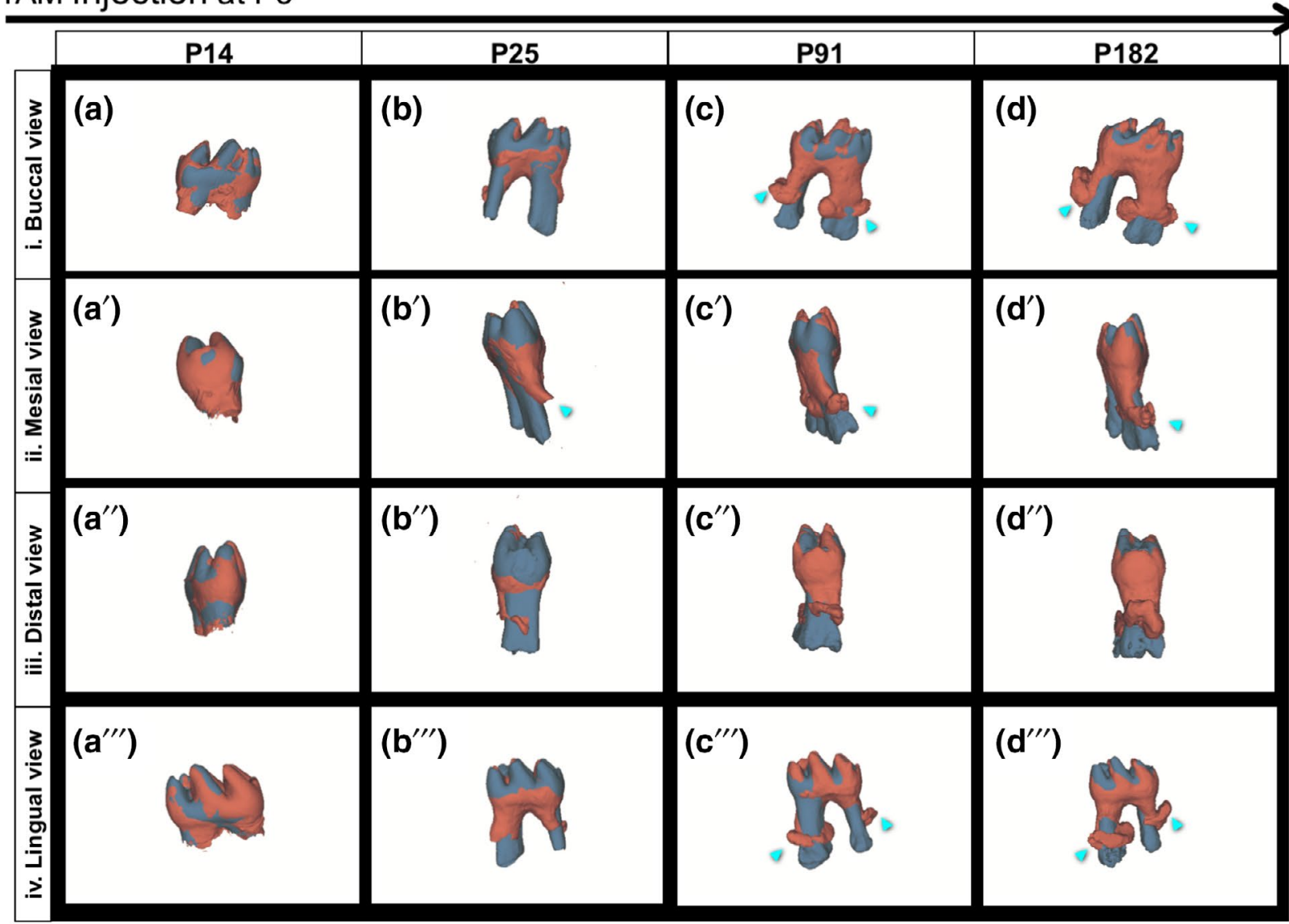

(DF-PPR)-cHET TEETH

Mesio-Distal Crown Length

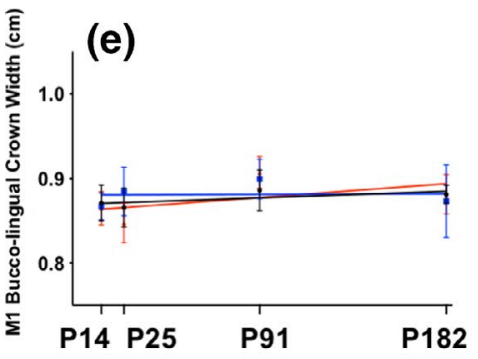

\section{Bucco-Lingual Crown Width}

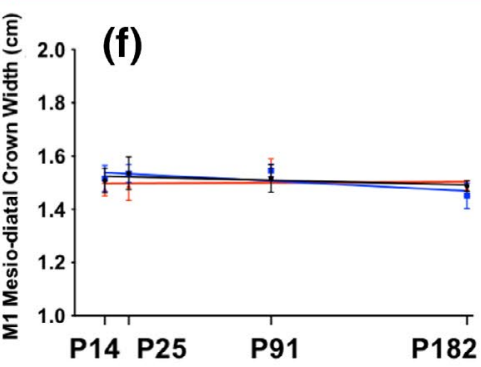

first Molar Root Length

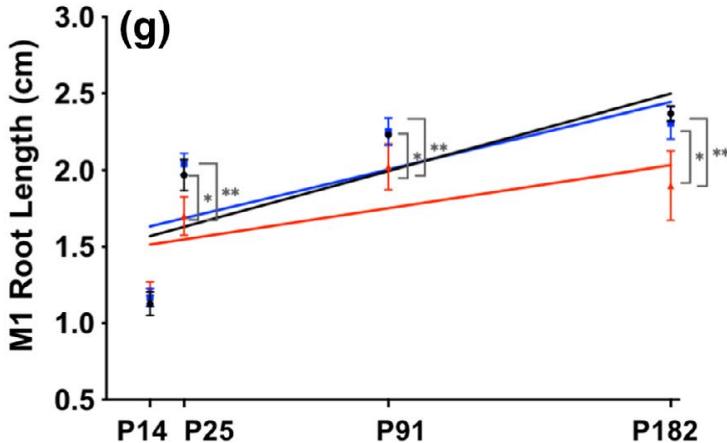

FIGURE 3 Mouse primary failure of eruption (PFE) molars show shorter and dilacerated roots. (a-d") Composite 3D surface model overlay of the mandibular first molars (M1), views from the buccal (a-d), mesial (a'-d'), distal (a"-d") and lingual (a'"'-d'") side (DF-PPR) cHet and (DF-PPR) cKO M1 were superimposed on the crown. Dark gray: (DF-PPR) cHet, red: (DF-PPR) cKO. Blue arrowheads: truncation (short roots) associated with dilacerations (curved roots). (e-g) Quantitative 3D- $\mu$ CT analysis. M1 mesiodistal crown length (e), buccolingual crown width $(f)$ and root length $(g)$ measured on 3D- $\mu C T$ images. Colored lines: regression line, black: Control, blue: (DF-PPR) cHet, red: (DF-PPR) cKO. At P14 $n=5$, P25 $n=6$, P91 and P182 $n=4{ }^{* *} p<.01,{ }^{*} p<.05$, One-way ANOVA followed by Mann-Whitney's U test. All data are represented as mean $\pm S D$

\section{DISCUSSION}

In this study, we reveal the pathophysiology of PFE conditions in a chronological manner, which expands from the initial stage of pre-emergence to postemergence tooth eruption, using a mouse model of PFE and detailed 3D microCT analyses. These sequential events in mice correspond to the preadolescence, the adolescence, the adulthood, and the aging stage of life in humans. Our data indicate that mouse cKO molars have a statistically significant delay in eruption, root malformation, and defective formation of the interradicular bone. Interestingly and strikingly, no cessation of tooth eruption in PFE molars is observed unlike previously postulated, although tooth eruption continues at a much slower rate than that of other two groups. As a result, the difference in the eruption 
Axial section of mandible

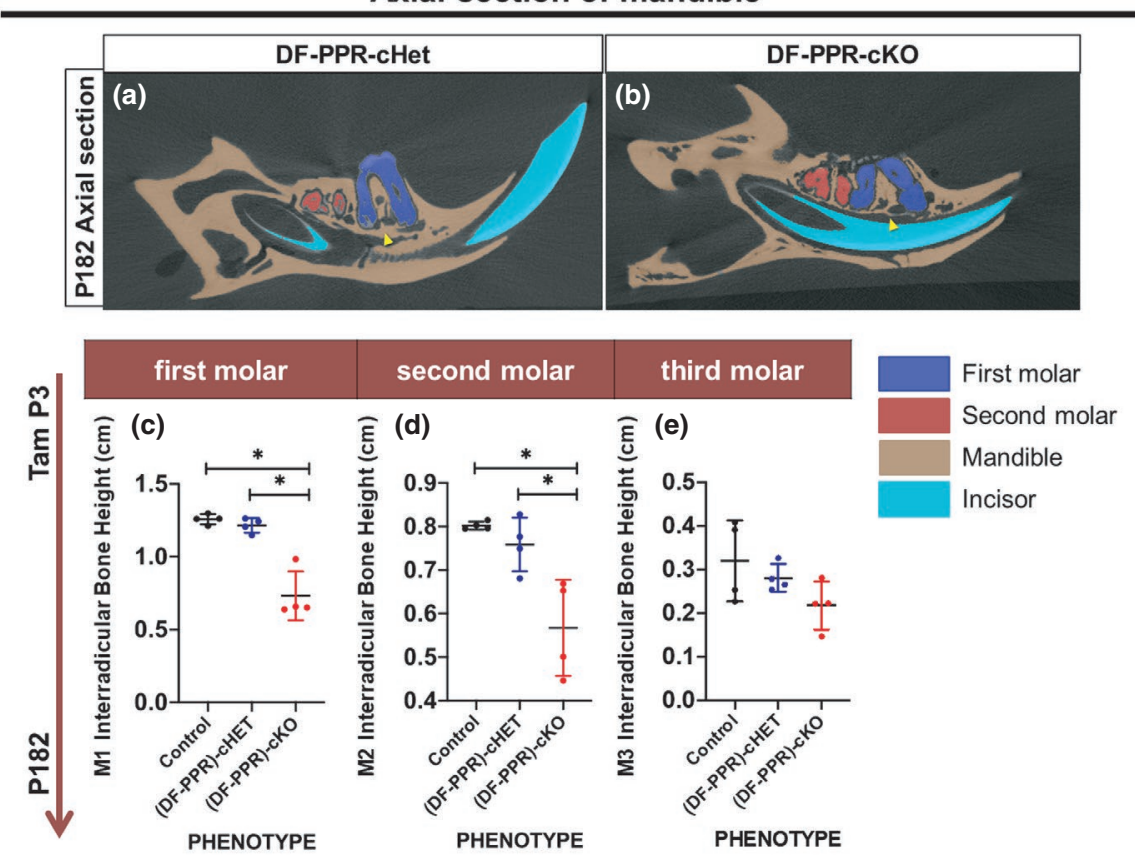

FIGURE 4 Mouse primary failure of eruption (PFE) molars defective cryptal bone formation. (a,b) Axial sections of 3D- $\mu$ CT images, (DF-PPR) cHet (a) and (DF-PPR) CKO (b) at P182. Blue: M1, red: $M 2$, beige: mandible, blue: incisor. Arrowheads: interradicular cryptal bone. (c-e) Quantitative 3D- $\mu C T$ analysis. Interradicular bone height of M1 (c), M2 (d) and M3 (e) measured on 3D- $\mu \mathrm{CT}$ images. ${ }^{*} p<.05$, One-way ANOVA followed by Mann-Whitney's U test. All data are represented as mean $\pm S D$ height between cKO first molars and those of the control group increases overtime. The second molars, on the other hand, seem to have the same eruption rate as the Control, but still exhibit a statistically significant less eruption height. These data are consistent with clinical features of type II human PFE in which first molars are usually more affected than second molars (Frazier-Bowers et al., 2007). However, additional studies are needed to determine the mechanism underlying these phenotypes. In addition, coronal superimposition images show that cKO molars unanimously have truncated and dilacerated roots. These morphological root anomalies of mouse PFE molars are reminiscent of those observed in human PFE molars, which are associated with tooth root abnormality and normal crown morphology (Cohen-Lévy \& Cohen, 2015; Deffrennes \& Cohen-Lévy, 2015; Pilz et al., 2014). Furthermore, there is a significant reduction of the interradicular bone height in cKO first and second molars at P182, indicating defective bone formation in this specific area. As phenotypes in mouse PPR cKO molars mostly recapitulate human PFE conditions, we think that this mouse model is a useful tool for studying tooth eruption and root formation associated with PFE. However, additional human subjects are needed in order to establish a firm correlation between mouse and human PFE conditions.

Of note, the difference in the eruption height and the root length becomes increasingly pronounced after $\mathrm{P} 25$, when formation of the periodontal attachment apparatus, that is, PDL, cementum and surrounding alveolar bone, is complete. Disruption in the PPR signaling in PTHrP-expressing dental follicle cells results in lack of periodontal ligament, malformed cementum, and defective alveolar cryptal bone, which lead to defective eruption process. These findings also serve as evidence of the importance of tooth root and periodontal attachment apparatus formation during the emergence and the postemergence stage of tooth eruption.
Our finding is intriguingly reminiscent of the previous longterm follow-up case report of human PFE, which demonstrates the aggravation of open bite in PFE patients in adulthood (Kanno et al., 2017). The mouse PFE molars also demonstrate similar aggravation of the eruption height, particularly of the first molars. The quantitative evaluation of microCT is a reliable method for the linear measurement (Kim, Paik, \& Lee, 2007). By utilizing more detailed 3D microCT analyses, our findings identify that, instead of cessation of the tooth eruption, this PFE phenotype is largely attributed to the slower rate of tooth eruption. Nevertheless, more investigation is needed to identify the cause of aggravating open bite conditions in human PFE.

Even though human and mice PFE shares the similar phenotypes, the difference in genetic conditions should be considered. While in human PFE is caused by loss-of-function mutations in PTHR1 gene (Decker et al., 2008; Frazier-Bowers et al., 2014, 2010; Grippaudo et al., 2018; Jelani et al., 2016; Risom et al., 2013; Roth et al., 2014; Yamaguchi et al., 2011), mouse PFE is caused by the artificial genetic manipulation in which PPR is specifically deleted in a population of PTHrP-expressing mesenchymal progenitors of the dental follicle (Takahashi et al., 2019). Therefore, the actual mechanism underlying human PFE might be different from that of mouse PFE. Further studies of each human PFE receptor mutations are needed to fully understand the pathophysiology of human PFE.

\section{5 | CONCLUSIONS}

The chronology of tooth eruption and root formation of mouse PFE molars might correspond to that of human PFE molars, with respect to delayed eruption, tooth root abnormal morphology, and decreased interradicular bone height. 


\section{ACKNOWLEDGEMENTS}

This study was supported by American Association of Orthodontists Foundation Grant to W.O., National Institution of Health Grants Grant DE027421 to W.O. and DE026666 to N.O., and University of Michigan MCubed 2.0 Grant to W.O. and N.O. Conflicts of interest: none to declare.

\section{ORCID}

Tetsutaro Yamaguchi (iD https://orcid.org/0000-0001-9806-7163 Wanida Ono iD https://orcid.org/0000-0002-0358-1897

\section{REFERENCE}

Baccetti, T. (2000). Tooth anomalies associated with failure of eruption of first and second permanent molars. American Journal of Orthodontics and Dentofacial Orthopedics, 118(6), 608-610. https:// doi.org/10.1067/mod.2000.97938

Cevidanes, L. H., Styner, M., \& Proffit, W. R. (2009). Three-dimensional superimposition of the skull base for the longitudinal evaluation of the effects of growth and of treatment. Orthodontie Francaise, 80(4), 347-357. https://doi.org/10.1051/orthodfr/2009021

Cohen-Lévy, J., \& Cohen, N. (2015). Eruption abnormalities in permanent molars: Differential diagnosis and radiographic exploration. Journal of Dentofacial Anomalies and Orthodontics, 18(4), 403. https://doi. org/10.1051/odfen/2014054

Dean, T., Vilardaga, J. P., Potts, J. T., \& Gardella, T. J. (2008). Altered selectivity of parathyroid hormone (PTH) and PTH-related protein (PTHrP) for distinct conformations of the PTH/PTHrP receptor. Molecular Endocrinology, 22(1), 156-166. https://doi.org/10.1210/ me.2007-0274

Decker, E., Stellzig-Eisenhauer, A., Fiebig, B. S., Rau, C., Kress, W., Saar, K., ... Weber, B. H. (2008). PTHR1 loss-of-function mutations in familial, nonsyndromic primary failure of tooth eruption. American Journal of Human Genetics, 83(6), 781-786. https://doi.org/10.1016/j. ajhg.2008.11.006

Deffrennes, D., \& Cohen-Lévy, J. (2015). Primary Failure of Eruption (PFE) and ankylosis of permanent molars: The surgeon's experience. Journal of Dentofacial Anomalies and Orthodontics, 18(4), 407. https:// doi.org/10.1051/odfen/2015022

Frazier-Bowers, S. A., Hendricks, H. M., Wright, J. T., Lee, J., Long, K., Dibble, C. F., \& Bencharit, S. (2014). Novel mutations in PTH1R associated with primary failure of eruption and osteoarthritis. Journal of Dental Research, 93(2), 134-139. https://doi.org/10.1177/00220 34513513588

Frazier-Bowers, S. A., Koehler, K. E., Ackerman, J. L., \& Proffit, W. R. (2007). Primary failure of eruption: Further characterization of a rare eruption disorder. American Journal of Orthodontics and Dentofacial Orthopedics, 131(5), 578.e571-511. https://doi.org/10.1016/j. ajodo.2006.09.038

Frazier-Bowers, S. A., Simmons, D., Wright, J. T., Proffit, W. R., \& Ackerman, J. L. (2010). Primary failure of eruption and PTH1R: The importance of a genetic diagnosis for orthodontic treatment planning. Am J Orthod Dentofacial Orthop, 137(2), 160.e161-167; discussion. https://doi.org/10.1016/j.ajodo.2009.10.019

Grippaudo, C., Cafiero, C., D'Apolito, I., Ricci, B., \& Frazier-Bowers, S. A. (2018). Primary failure of eruption: Clinical and genetic findings in the mixed dentition. Angle Orthodontist, 88(3), 275-282. https://doi. org/10.2319/062717-430.1

Honda, M. J., Imaizumi, M., Tsuchiya, S., \& Morsczeck, C. (2010). Dental follicle stem cells and tissue engineering. Journal of Oral Science, 52(4), 541-552. https://doi.org/10.2334/josnusd.52.541

Jelani, M., Kang, C., Mohamoud, H. S. A., Al-Rehaili, R., Almramhi, M. M., Serafi, R., ... Alkhiary, Y. M. (2016). A novel homozygous PTH1R variant identified through whole-exome sequencing further expands the clinical spectrum of primary failure of tooth eruption in a consanguineous Saudi family. Archives of Oral Biology, 67, 28-33. https://doi. org/10.1016/j.archoralbio.2016.03.012

Jüppner, H., Abou-Samra, A. B., Freeman, M., Kong, X. F., Schipani, E., Richards, J., ... Kronenberg, H. M. (1991). A G protein-linked receptor for parathyroid hormone and parathyroid hormone-related peptide. Science, 254(5034), 1024-1026.

Kanno, C. M., de Oliveira, J. A., Garcia, J. F., Roth, H., \& Weber, B. H. (2017). Twenty-year follow-up of a familial case of PTH1R-associated primary failure of tooth eruption. American Journal of Orthodontics and Dentofacial Orthopedics, 151(3), 598-606. https://doi.org/10.1016/j. ajodo.2016.09.012

Kim, I., Paik, K. S., \& Lee, S. P. (2007). Quantitative evaluation of the accuracy of micro-computed tomography in tooth measurement. Clinical Anatomy, 20(1), 27-34. https://doi.org/10.1002/ca.20265

Kjær, I. (2014). Mechanism of human tooth eruption: Review article including a new theory for future studies on the eruption process. Scientifica (Cairo), 2014, 341905. https://doi.org/10.1155/2014/341905

Kobayashi, T., Chung, U. I., Schipani, E., Starbuck, M., Karsenty, G., Katagiri, T., ... Kronenberg, H. M. (2002). PTHrP and Indian hedgehog control differentiation of growth plate chondrocytes at multiple steps. Development, 129(12), 2977-2986.

Massler, M., \& Schour, I. (1941). Studies in tooth development: Theories of eruption. American Journal of Orthodontics and Oral Surgery, 27(10), 552-557. https://doi.org/10.1016/S0096-6347(41)90413-1

Mizuhashi, K., Ono, W., Matsushita, Y., Sakagami, N., Takahashi, A., Saunders, T. L., ... Ono, N. (2018). Resting zone of the growth plate houses a unique class of skeletal stem cells. Nature, 563(7730), 254258. https://doi.org/10.1038/s41586-018-0662-5

Nagata, M., Ono, N., \& Ono, W. (2019). Mesenchymal progenitor regulation of tooth eruption: A view from PTHrP. Journal of Dental Research, 22034519882692. https://doi.org/10.1177/0022034519882692

Nanci, A. (2017). Ten Cate's Oral Histology-e-book: development, structure, and function, 9th ed. St.Louis (MO): Elsevier Health Sciences.

Ono, W., Sakagami, N., Nishimori, S., Ono, N., \& Kronenberg, H. M. (2016). Parathyroid hormone receptor signalling in osterix-expressing mesenchymal progenitors is essential for tooth root formation. Nature Communications, 7, 11277. https://doi.org/10.1038/ncomm s11277

Pilz, P., Meyer-Marcotty, P., Eigenthaler, M., Roth, H., Weber, B. H., \& Stellzig-Eisenhauer, A. (2014). Differential diagnosis of primary failure of eruption (PFE) with and without evidence of pathogenic mutations in the PTHR1 gene. Journal of Orofacial Orthopedics / Fortschritte der Kieferorthopädie, 75(3), 226-239. https://doi. org/10.1007/s00056-014-0215-y

Proffit, W. R., \& Frazier-Bowers, S. A. (2009). Mechanism and control of tooth eruption: Overview and clinical implications. Orthodontics and Craniofacial Research, 12(2), 59-66. https://doi. org/10.1111/j.1601-6343.2009.01438.x

Proffit, W. R., \& Vig, K. W. (1981). Primary failure of eruption: A possible cause of posterior open-bite. American Journal of Orthodontics, 80(2), 173-190. https://doi.org/10.1016/0002-9416(81)90217-7

Raghoebar, G. M., Boering, G., Vissink, A., \& Stegenga, B. (1991). Eruption disturbances of permanent molars: A review. Journal of Oral Pathology and Medicine, 20(4), 159-166. https://doi. org/10.1111/j.1600-0714.1991.tb00913.x

Richman, J. M. (2019). Shedding new light on the mysteries of tooth eruption. Proceedings of the National Academy of Sciences, 116(2), 353-355. https://doi.org/10.1073/pnas.1819412116

Risom, L., Christoffersen, L., Daugaard-Jensen, J., Hove, H. D., Andersen, H. S., Andresen, B. S., ... Duno, M. (2013). Identification of six novel PTH1R mutations in families with a history of primary failure of tooth eruption. PLoS ONE, 8(9), e74601. https://doi.org/10.1371/journ al.pone.0074601 
Roth, H., Fritsche, L. G., Meier, C., Pilz, P., Eigenthaler, M., MeyerMarcotty, P., ... Weber, B. H. F. (2014). Expanding the spectrum of PTH1R mutations in patients with primary failure of tooth eruption. Clin Oral Investig, 18(2), 377-384. https://doi.org/10.1007/ s00784-013-1014-3

Ruellas, A. C. D. O., Tonello, C., Gomes, L. R., Yatabe, M. S., Macron, L., Lopinto, J., ... Cevidanes, L. H. S. (2016). Common 3-dimensional coordinate system for assessment of directional changes. American Journal of Orthodontics and Dentofacial Orthopedics, 149(5), 645-656. https://doi.org/10.1016/j.ajodo.2015.10.021

Ruellas, A. C. D. O., Yatabe, M. S., Souki, B. Q., Benavides, E., Nguyen, T., Luiz, R. R., ... Cevidanes, L. H. S. (2016). 3D mandibular superimposition: Comparison of regions of reference for voxel-based registration. PLoS ONE, 11(6), e0157625. https://doi.org/10.1371/journ al.pone.0157625

Takahashi, A., Nagata, M., Gupta, A., Matsushita, Y., Yamaguchi, T., Mizuhashi, K., ... Ono, W. (2019). Autocrine regulation of mesenchymal progenitor cell fates orchestrates tooth eruption. Proceedings of the National Academy of Sciences, 116(2), 575-580. https://doi. org/10.1073/pnas.1810200115

Wise, G. E. (2009). Cellular and molecular basis of tooth eruption. Orthodontics and Craniofacial Research, 12(2), 67-73. https://doi. org/10.1111/j.1601-6343.2009.01439.x
Wise, G. E., Frazier-Bowers, S., \& D'Souza, R. N. (2002). Cellular, molecular, and genetic determinants of tooth eruption. Critical Reviews in Oral Biology and Medicine, 13(4), 323-334.

Yamaguchi, T., Hosomichi, K., Narita, A., Shirota, T., Tomoyasu, Y., Maki, K., \& Inoue, I. (2011). Exome resequencing combined with linkage analysis identifies novel PTH1R variants in primary failure of tooth eruption in Japanese. Journal of Bone and Mineral Research, 26(7), 1655-1661. https://doi.org/10.1002/jbmr.385

\section{SUPPORTING INFORMATION}

Additional supporting information may be found online in the Supporting Information section.

FigS1FigS2

How to cite this article: Tokavanich N, Gupta A, Nagata M, et al. A three-dimensional analysis of primary failure of eruption in humans and mice. Oral Dis. 2020;26:391-400. https://doi.org/10.1111/odi.13249 